\title{
How to Outsource Coaching in School-Based Extracurricular Sports Activities: Evaluating Perceptions of External Coaches
}

\author{
Kenryu Aoyagi ${ }^{1,2, *}$, Kaori Ishii ${ }^{3}$, Ai Shibata ${ }^{4}$, Hirokazu Arai ${ }^{5} \&$ Koichiro Oka $^{3}$ \\ ${ }^{1}$ Graduate School of Sport Sciences, Waseda University, 2-579-15 Mikajima Tokorozawa, \\ Saitama 359-1192, Japan \\ ${ }^{2} J a p a n$ Society for the Promotion of Science, 5-3-1 Koujimachi Chiyoda, Tokyo 102-0083, \\ Japan \\ ${ }^{3}$ Faculty of Sport Sciences, Waseda University, 2-579-15 Mikajima Tokorozawa, Saitama \\ 359-1192, Japan \\ ${ }^{4}$ Faculty of Health and Sport Sciences, University of Tsukuba, 1-1-1 Tennodai, Tsukuba, \\ Ibaraki 305-8574, Japan \\ ${ }^{5}$ Faculty of Letters, Hosei University, 2-17-1 Fujimi Chiyoda, Tokyo 102-8160, Japan \\ *Corresponding author: Graduate School of Sport Sciences, Waseda University, 2-579-15 \\ Mikajima Tokorozawa, Saitama 359-1192, Japan
}

Received: July 8, 2014

doi:10.5296/ije.v6i3.5942
Accepted: August 5, 2014 Published: September 1, 2014

URL: http://dx.doi.org/10.5296/ije.v6i3.5942

\begin{abstract}
Participating in school-based extracurricular sports activities is valuable for the healthy development of children and adolescents. The use of external coaches (outsourcing human resources) has been suggested to support teachers in coaching and managing such activities. However, hiring external coaches is difficult, and their number is insufficient. The purposes of the present study were therefore to evaluate the facilitators and barriers for engaging external coaches in school-based extracurricular sports activities, and determine whether differences in facilitators and barriers exist for the coaches and if these are related to the various characteristics of the coaches. A cross-sectional self-administrated questionnaire was distributed to 1974 external coaches, who indicated whether they felt each facilitator and barrier applied to them with respect to engaging in school-based extracurricular sports activities. Data were obtained from 149 coaches, and they perceived many facilitators and barriers. Some differences were found in the responses related to the coaches'
\end{abstract}




\section{Macrothink}

International Journal of Education

ISSN 1948-5476

2014, Vol. 6, No. 3

characteristics - especially with respect to age-group and their willingness or unwillingness to become full-time teachers. In conclusion, the following steps would be valuable toward creating an effective strategy to promote the engagement of external coaches in school-based extracurricular sports activities: improving the coaching environment for the coaches such that they feel enjoyment or undergo personal development; easing institutional limitations; enhancing teacher support and participation in the activities; allotting appropriate roles for the teacher and external coach; and holding activity workshops.

Keywords: extracurricular activity, human resource management, mixed methods approach, physical education, volunteer 


\section{Introduction}

Participating in sports is valuable for a healthy, culturally rich life [Ministry of Education, Culture, Sports, Science and Technology in Japan (MEXT), 2011]. In view of the carry-over effect of physical activity from childhood to adulthood (Telama, 2009), it is important to sustain an environment in which children and adolescents can be physically active. Physical education in the school curriculum provides an opportunity to participate in sports and physical activity. Though the number of classes devoted to physical education is limited (MEXT, 2008, 2009), school-based extracurricular sports activities (SBECSA) - conducted after school or on weekends - offer other opportunities for participation. In addition, it is easier to take part in SBECSA than in community sports owing to the costs, lack of transportation needs, and the familiar setting (Meester, Aelterman, Cardon, Bourdeaudhuij, \& Haerens, 2014). Indeed, many SBECSA are performed on the school setting after school or on weekends (Australian Bureau of Statistics, 2012; Edwards, Kanters, \& Bocarro, 2011; MEXT, 2013; Sport Council Wales, 2009). Engaging in SBECSA contributes not only to the physical development but also to the mental, academic, and social development of adolescents (Barnett, 2007; Dotterer, McHale, \& Crouter, 2007; Fredricks \& Eccles, 2006; Lipscomb, 2007; MEXT, 2012; Schaefer, Simpkins, Vest, \& Price, 2011; Shernoff \& Vandell, 2007). As a result of these benefits, SBECSA should be actively encouraged for their positive merits.

In Japan, the use of external coaches (outsourcing human resources) has been suggested as a way of supporting teachers in the coaching and management of SBECSA (MEXT, 2013). Owing to the lack of properly trained teachers able to coach SBECSA (Yamagata Prefecture Board of Education, 2010), the transfer of SBECSA teachers to other schools leading to a reduction in SBECSA at the original school (Tokyo Metropolitan Board of Education, 2007), and considerable workload burdens for teachers to manage the SBECSA (Japan Senior High School Teachers and Staff Union, 2008; MEXT, 1997; Whiteley \& Richard, 2012), over 80\% of school principals and teachers in Japan are in favor of the use of external coaches for SBECSA (MEXT, 1997). Furthermore, the benefits of using external coaches include an increase in students' interest and participation in SBECSA and improvement in the coaching skills of teachers that manage the activities (Tokyo Metropolitan Board of Education, 2008). However, the difficulties in hiring external coaches (Aoyagi et al., 2013a; Miyagi Prefecture Board of Education, 2008; Williams, Hay, \& Macdonald, 2011; Yamagata Prefecture Board of Education, 2010) and the small number of coaches in some regions of Japan and for certain types of sport have been reported (Nippon Junior High School Physical Culture Association, 2013; Nishijima, Yano, \& Nakazawa, 2007). Additionally, the number of coaching hours provided by external coaches is insufficient to meet demand (Miyagi Prefecture Board of Education, 2008; Yamagata Prefecture Board of Education, 2010). Thus, enhancing the engagement of external coaches - number of coaches, coaching frequency, and continuous participation-is important for the teachers who need their help.

Enhancing the engagement of external coaches for SBECSA demands the development of effective strategies (MEXT, 2013). Previous surveys and studies have attempted to clarify the facilitators and barriers related to the engagement of external SBECSA coaches (Kanagawa 
Prefecture Board of Education, 2008; LaVoi \& Dutove, 2012; Miyagi Prefecture Board of Education, 2008). However, most of that research was conducted with a limited number of questions and limited sample groups; thus, those facilitators and barriers have been only partially examined. Using semi-structured interviews, Aoyagi et al. (2013c) qualitatively identified categories of facilitators and barriers for engaging external SBECSA coaches; they included system, support, environment, and climate of SBECSA. However, the extent to which each facilitator and barrier related to the engagement of those coaches was not addressed. Additionally, it is unclear whether the facilitators and barriers depend on various characteristics of the coaches. Thus, the present study aimed to examine the importance of the facilitators and barriers to the engagement of external SBECSA coaches, as initially investigated in a previous qualitative study (Aoyagi et al., 2013c); it also had the objective of determining whether differences in facilitators and barriers exert different effects according to the personal characteristics of such coaches.

\section{Methods}

\subsection{Participants and Procedures}

A cross-sectional self-administrated questionnaire survey was given to external SBECSA coaches at public junior high and high schools. Request letters for study participation along with a set of questionnaires (including instructions and a consent form) were sent to the principals of 658 randomly selected schools (329 junior high and 329 high schools). All of Japan's 47 prefectures were represented, and seven junior high and seven high schools were selected from each prefecture. Combined junior high and high schools, evening schools, and branch schools were excluded before the random sampling because they are minority in the school system and may have biased the results. Based on the average number of external coaches (Sasakawa Sports Foundation, 2011; Tokyo Metropolitan Board of Education, 2008), three sets of the questionnaires were sent to each school. The maximum numbers of participants was therefore 1974 . The school principal distributed the questionnaires to the SBECSA teachers, and they in turn gave the questionnaires to the external coaches. Upon completion, the surveys were returned to the researchers. This survey was conducted in 2012 . The study purpose and design were explained to the participants, and they all provided their written informed consent. The research proposal was approved by the ethics board of Waseda University (No. 2011-242).

\subsection{Questionnaire}

The questionnaire contained 53 questions to assess perceived facilitators and 54 questions to determine perceived barriers to engaging in SBECSA for the external coaches. The questions covered all the facilitators and barriers reported by external coaches in a previous study, which included interviews, and extracted using qualitative analysis (Aoyagi et al., 2013c). The facilitators were constructed with large categories, such as positive feelings, adequate system, positive social support, comfortable climate of SBECSA, environment, growth of external coach, and network building. The barriers consisted of such categories as negative 


\section{Macrothink}

feelings, inadequate system, lack of support, uncomfortable climate of SBECSA, poor environment, and burdens. The responses were set on a six-point scale, ranging from "not applicable at all" (0) to "very applicable" (5). Each participant was also asked to complete a series of sociodemographic questions about their gender, age, and the type of school where they coached (junior high school or high school). They were also asked whether or not they were willing to become a full-time teacher.

\subsection{Analyses}

To estimate the importance of each facilitator and barrier, the percentages of applicability were calculated. Scores of 0-2 were defined as inapplicable answers and 3-5 were considered as applicable. The percentages of applicability between gender (male or female), age (younger or older group dichotomized by mean age), type of school (junior high or high school), and external coaches who were and were not willing to become full-time teachers were calculated respectively. Then, chi-square tests $(\alpha=.05)$ were conducted to verify the differences between the two groups for each facilitator and barrier. Any missing values were excluded. All analyses were performed using IBM SPSS Statistics Version 21.

\section{Results}

\subsection{Participant Characteristics}

Responses were received from 149 external coaches (response rate, 7.5\%). The participant characteristics appear in Table 1. There were 129 male (86.6\%) and 20 female (13.4\%). The participants showed a wide age-range, with a mean age of 46.7 years (standard deviation, 15.1). The numbers of external coaches who coached at junior high and high schools were 86 $(57.7 \%)$ and 59 (39.6\%), respectively. Seventeen external coaches were willing to become full-time teachers, 12 of whom were in their $20 \mathrm{~s}$. 
Table 1. Participant characteristics

\begin{tabular}{lrr}
\hline & $\mathrm{n}$ & $\%$ \\
\hline Overall & 149 & 100.0 \\
Gender & & \\
Male & 129 & 86.6 \\
Female & 20 & 13.4 \\
Missing & 0 & 0.0 \\
Age group & & \\
20-29 & 27 & 18.1 \\
$30-39$ & 24 & 16.1 \\
$40-49$ & 33 & 22.1 \\
$50-59$ & 33 & 22.1 \\
$60-69$ & 19 & 12.8 \\
$70-80$ & 12 & 8.1 \\
Missing & 1 & 0.7 \\
Mean \pm SD & & $46.7 \pm 15.1$ \\
Type of school & & \\
Junior high school & 86 & 57.7 \\
High school & 59 & 39.6 \\
Missing & 4 & 2.7 \\
Willing to be a teacher & & \\
Yes & 17 & 11.4 \\
No & 123 & 82.6 \\
Missing & 9 & 6.0 \\
\hline
\end{tabular}

\subsection{Facilitators to Engaging in SBECSA}

For 39 of the 53 facilitators, more than $50 \%$ of the external coaches indicated that they were applicable (Table 2). The facilitators that were reported to be applicable by over $90 \%$ of the coaches were as follows: $\mathrm{f} 2$, feeling enjoyment (98.6\%); f5, feeling enjoyment to consider team members $(91.3 \%)$; f12, love of the sport that external coach plays $(100.0 \%)$; f16, desire for team members to win (94.6\%); f22, having a cooperative SBECSA teacher $(91.3 \%)$; f30, seeing technical improvement in team members $(98.0 \%)$; 31 , seeing personal progress in team members $(96.0 \%)$; f36, having a coaching environment available (95.3\%); and f40, learning of external coach $(92.6 \%)$. Within the large category of comfortable climate of SBECSA, all the small categories were considered applicable by over $80 \%$ of the coaches. Financial compensation was not regarded as an important facilitator (12.2\%).

Only one facilitator- $\mathrm{f} 25$, understanding from parents - was reported more frequently as a facilitator among female than male $(100.0 \%$ and $79.5 \%$, respectively). Four items showed a significantly higher rate of applicable responses from coaches in the younger (20-46 years) than older age-group (47-80 years old): $\mathrm{f37}$, having a SBECSA teacher to learn from $(54.3 \%$ versus $33.8 \%$ ); f49, being able to network (75.7\% versus $51.3 \%)$; f50, increasing acquaintances $(77.1 \%$ versus $61.5 \%)$; and 553 , getting a position as a part-time teacher $(10.0 \%$ versus $1.3 \%$ ). Subjects who coached in junior high schools felt that one item-f24, 
understanding from teachers other than the SBECSA teacher-was more applicable than those who coached in high schools $(80.2 \%$ versus $63.8 \%)$; participants who coached in high schools felt that two items-f19, getting compensation $(19.0 \%$ versus $7.0 \%)$ and $f 23$, adjusting practice time around external coach's schedule (55.9\% versus $32.6 \%)$-were more applicable than those coaching in junior high schools.

With five items, there were higher proportions among coaches who were willing to become full-time teachers: $\mathrm{f37}$, having a SBECSA teacher to learn from (70.6\% versus $39.3 \%)$; $\mathrm{f} 47$, accumulating coaching experience $(88.2 \%$ versus $61.8 \%)$; f49, being able to network $(94.1 \%$ versus 58.5\%); f50, increasing acquaintances (94.1\% versus $65.0 \%$ ); and $\mathrm{f} 53$, getting a position as a part-time teacher $(17.6 \%$ versus $3.3 \%)$. By contrast, with four items there were higher proportions among the coaches who did not wish to become full-time teachers: f13, vitalizing the local sport the external coach plays $(85.2 \%$ versus $58.8 \%)$; f20, being requested by the organization to which external coach belongs (33.6\% versus $5.9 \%)$; f33, alma mater of external coach $(41.0 \%$ versus $11.8 \%)$; and f34, close proximity of school $(64.2 \%$ versus $35.3 \%)$.

Table 2. Percentages for facilitators of involvement among the external coaches

\begin{tabular}{|c|c|c|c|c|c|c|c|}
\hline \multirow{2}{*}{$\begin{array}{l}\text { Large } \\
\text { category (7) }\end{array}$} & \multirow{2}{*}{$\begin{array}{l}\text { Middle category } \\
\text { (24) }\end{array}$} & \multirow[t]{2}{*}{ Small category (53) } & \multirow[t]{2}{*}{ Overall } & \multicolumn{2}{|c|}{ Age group } & \multicolumn{2}{|c|}{$\begin{array}{l}\text { Willing to be a } \\
\text { teacher }\end{array}$} \\
\hline & & & & $20-46$ & $47-80$ & Yes & No \\
\hline \multirow{16}{*}{$\begin{array}{l}\text { positive } \\
\text { feelings }\end{array}$} & \multirow{6}{*}{ enjoyment } & f1. sharing a dream with team members & 82.6 & 82.9 & 82.1 & 82.4 & 81.3 \\
\hline & & f2. feeling enjoyment & 98.6 & 98.6 & 98.7 & 100.0 & 98.4 \\
\hline & & f3. having a refreshing change & 53.7 & 60.9 & 47.4 & 52.9 & 52.9 \\
\hline & & f4. achieving a sense of fulfillment & 85.9 & 82.9 & 88.5 & 82.4 & 85.4 \\
\hline & & $\begin{array}{l}\text { f5. feeling enjoyment to consider team } \\
\text { members }\end{array}$ & 91.3 & 90.0 & 92.3 & 100.0 & 89.4 \\
\hline & & f6. having fun as a coach & 40.3 & 45.7 & 35.9 & 47.1 & 37.4 \\
\hline & \multirow{3}{*}{$\begin{array}{l}\text { parental-like } \\
\text { warmth toward } \\
\text { team members }\end{array}$} & $\begin{array}{l}\text { f7. feeling parental-like warmth toward } \\
\text { team members }\end{array}$ & 87.9 & 88.6 & 87.2 & 76.5 & 88.6 \\
\hline & & $\begin{array}{l}\text { f8. desire to continue to involve team } \\
\text { members }\end{array}$ & 89.3 & 92.9 & 85.9 & 88.2 & 88.6 \\
\hline & & $\begin{array}{l}\text { f9. desire to help team members } \\
\text { continue to engage in SBECSA }\end{array}$ & 88.5 & 87.1 & 89.6 & 94.1 & 86.9 \\
\hline & \multirow[b]{2}{*}{ desire to coach } & f10. desire to coach & 87.2 & 91.4 & 83.3 & 88.2 & 86.2 \\
\hline & & $\begin{array}{l}\text { f11. desire to have a positive influence } \\
\text { as a coach }\end{array}$ & 63.1 & 58.6 & 66.7 & 58.8 & 62.6 \\
\hline & \multirow{2}{*}{ love for the sport } & $\begin{array}{l}\text { f12. love of the sport that external } \\
\text { coach plays }\end{array}$ & 100.0 & 100.0 & 100.0 & 100.0 & 100.0 \\
\hline & & $\begin{array}{l}\text { f13. vitalizing the local sport the } \\
\text { external coach plays }\end{array}$ & 82.4 & 81.4 & 83.1 & 58.8 & $85.2 *$ \\
\hline & \multirow{2}{*}{$\begin{array}{l}\text { commitment or } \\
\text { responsibility }\end{array}$} & $\begin{array}{l}\text { f14. having a commitment or } \\
\text { responsibility }\end{array}$ & 89.9 & 90.0 & 89.7 & 88.2 & 90.2 \\
\hline & & f15. desire to repay an obligation & 79.2 & 82.9 & 75.6 & 70.6 & 79.7 \\
\hline & desire to win & f16. desire for team members to win & 94.6 & 98.6 & 91.0 & 100.0 & 93.5 \\
\hline \multirow{5}{*}{$\begin{array}{l}\text { adequate } \\
\text { system }\end{array}$} & \multirow{2}{*}{ little responsibility } & $\begin{array}{l}\text { f17. affable status of external coach for } \\
\text { team members or their parents }\end{array}$ & 76.5 & 71.4 & 80.8 & 64.7 & 76.4 \\
\hline & & $\begin{array}{l}\text { f18. having little sense of responsibility } \\
\text { by getting no compensation }\end{array}$ & 10.8 & 12.9 & 9.1 & 11.8 & 10.7 \\
\hline & compensation & f19. getting compensation & 12.2 & 12.9 & 11.7 & 23.5 & 9.8 \\
\hline & $\begin{array}{l}\text { strong request from } \\
\text { an organization }\end{array}$ & $\begin{array}{l}\text { f20. being requested by the } \\
\text { organization to which external coach } \\
\text { belongs }\end{array}$ & 30.4 & 31.4 & 29.9 & 5.9 & $33.6^{*}$ \\
\hline & $\begin{array}{l}\text { rights of the } \\
\text { external coach }\end{array}$ & $\begin{array}{l}\text { f21. having the right to enter the } \\
\text { competition site }\end{array}$ & 46.6 & 39.1 & 52.6 & 56.3 & 43.8 \\
\hline
\end{tabular}




\begin{tabular}{|c|c|c|c|c|c|c|c|}
\hline \multirow{5}{*}{$\begin{array}{l}\text { positive } \\
\text { social } \\
\text { support }\end{array}$} & cooperative & $\begin{array}{l}\text { f22. having a cooperative SBECSA } \\
\text { teacher }\end{array}$ & 91.3 & 88.6 & 93.6 & 76.5 & 92.7 \\
\hline & SBECSA teacher & $\begin{array}{l}\text { f23. adjusting practice time around } \\
\text { external coach's schedule }\end{array}$ & 42.3 & 38.6 & 46.2 & 47.1 & 42.3 \\
\hline & $\begin{array}{l}\text { understanding from } \\
\text { the school }\end{array}$ & $\begin{array}{l}\text { f24. understanding from teachers other } \\
\text { than the SBECSA teacher }\end{array}$ & 74.3 & 77.1 & 71.4 & 70.6 & 73.8 \\
\hline & $\begin{array}{l}\text { understanding from } \\
\text { parents }\end{array}$ & f25. understanding from parents & 82.3 & 84.3 & 80.3 & 76.5 & 82.6 \\
\hline & cooperative family & f26. support from own family members & 62.4 & 55.7 & 67.9 & 58.8 & 61.8 \\
\hline \multirow{6}{*}{$\begin{array}{l}\text { comfortable } \\
\text { climate of } \\
\text { SBECSA }\end{array}$} & \multirow{3}{*}{$\begin{array}{l}\text { acceptance of } \\
\text { SBECSA }\end{array}$} & f27. acceptance of team members & 89.9 & 85.7 & 93.6 & 82.4 & 91.1 \\
\hline & & f28. respect from team members & 81.2 & 75.7 & 85.9 & 64.7 & 82.1 \\
\hline & & $\begin{array}{l}\text { f29. team members who take external } \\
\text { coach's advice }\end{array}$ & 85.9 & 81.4 & 89.7 & 82.4 & 85.4 \\
\hline & \multirow{2}{*}{$\begin{array}{l}\text { growth of team } \\
\text { members }\end{array}$} & $\begin{array}{l}\text { f30. seeing technical improvement in } \\
\text { team members }\end{array}$ & 98.0 & 98.6 & 97.4 & 100.0 & 98.4 \\
\hline & & $\begin{array}{l}\text { f31. seeing personal progress in team } \\
\text { members }\end{array}$ & 96.0 & 94.3 & 97.4 & 94.1 & 95.9 \\
\hline & $\begin{array}{l}\text { high motivation of } \\
\text { team members }\end{array}$ & f32. high motivation of team members & 86.5 & 90.0 & 83.1 & 76.5 & 87.7 \\
\hline \multirow{7}{*}{ environment } & \multirow{4}{*}{ easy access } & f33. alma mater of external coach & 38.5 & 41.4 & 35.9 & 11.8 & $41.0^{*}$ \\
\hline & & f34. close proximity of school & 61.1 & 55.7 & 66.7 & 35.3 & $64.2^{*}$ \\
\hline & & $\begin{array}{l}\text { f35. being able to go to practice facility } \\
\text { using only a commuter pass }\end{array}$ & 11.6 & 12.9 & 10.5 & 23.5 & 9.1 \\
\hline & & $\begin{array}{l}\text { f36. having a coaching environment } \\
\text { available }\end{array}$ & 95.3 & 92.9 & 97.4 & 88.2 & 95.9 \\
\hline & \multirow{2}{*}{$\begin{array}{l}\text { environment where } \\
\text { an external coach } \\
\text { can grow }\end{array}$} & $\begin{array}{l}\text { f37. having a SBECSA teacher to learn } \\
\text { from }\end{array}$ & 43.9 & $54.3^{*}$ & 33.8 & $70.6^{*}$ & 39.3 \\
\hline & & f38. being on a powerful team & 24.5 & 21.4 & 26.3 & 23.5 & 24.8 \\
\hline & $\begin{array}{l}\text { inadequate } \\
\text { environment }\end{array}$ & $\begin{array}{l}\text { f39. being motivated by a poor } \\
\text { SBECSA environment }\end{array}$ & 35.1 & 34.3 & 36.4 & 23.5 & 37.7 \\
\hline \multirow{9}{*}{$\begin{array}{l}\text { growth of } \\
\text { external } \\
\text { coach }\end{array}$} & \multirow{6}{*}{$\begin{array}{l}\text { growth of external } \\
\text { coach }\end{array}$} & f40. learning of external coach & 92.6 & 90.0 & 94.8 & 94.1 & 91.8 \\
\hline & & f41. growth of external coach & 83.8 & 87.1 & 80.5 & 88.2 & 82.0 \\
\hline & & $\begin{array}{l}\text { f42. enhancement of communication } \\
\text { skills }\end{array}$ & 85.8 & 82.6 & 88.5 & 82.4 & 85.2 \\
\hline & & $\begin{array}{l}\text { f43. learning ways to communicate } \\
\text { with team members }\end{array}$ & 74.3 & 81.4 & 67.5 & 94.1 & 71.3 \\
\hline & & $\begin{array}{l}\text { f44. enhancing competitive ability of } \\
\text { external coach }\end{array}$ & 63.1 & 65.7 & 60.3 & 64.7 & 61.8 \\
\hline & & $\begin{array}{l}\text { f45. enhancing physical fitness of } \\
\text { external coach }\end{array}$ & 59.1 & 55.7 & 61.5 & 47.1 & 60.2 \\
\hline & \multirow{3}{*}{$\begin{array}{l}\text { coaching } \\
\text { experience }\end{array}$} & f46. having an educational experience & 60.5 & 67.1 & 53.9 & 70.6 & 59.5 \\
\hline & & f47. accumulating coaching experience & 65.1 & 72.9 & 57.7 & $88.2 *$ & 61.8 \\
\hline & & $\begin{array}{l}\text { f48. talking with other coaches or } \\
\text { SBECSA teacher }\end{array}$ & 72.5 & 75.7 & 69.2 & 76.5 & 71.5 \\
\hline \multirow{5}{*}{$\begin{array}{l}\text { network } \\
\text { building }\end{array}$} & \multirow{3}{*}{ networking } & f49. being able to network & 63.1 & $75.7 * *$ & 51.3 & $94.1 * *$ & 58.5 \\
\hline & & f50. increasing acquaintances & 69.1 & $77.1 *$ & 61.5 & $94.1 *$ & 65.0 \\
\hline & & $\begin{array}{l}\text { f51. increasing customers for } \\
\text { self-employed external coaches }\end{array}$ & 4.7 & 1.4 & 7.8 & 0.0 & 5.7 \\
\hline & \multirow{2}{*}{$\begin{array}{l}\text { positive influence } \\
\text { on future career }\end{array}$} & $\begin{array}{l}\text { f52. perceiving that external coaching is } \\
\text { helpful in passing the teacher } \\
\text { adaptation examination }\end{array}$ & 13.8 & 15.7 & 12.2 & 29.4 & 11.7 \\
\hline & & $\begin{array}{l}\text { f53. getting a position as a part-time } \\
\text { teacher }\end{array}$ & 5.4 & $10.0 *$ & 1.3 & $17.6^{*}$ & 3.3 \\
\hline
\end{tabular}

Note. "f" placed in front of small category means "facilitator"; ** $=\mathrm{p}<.01 ; *=\mathrm{p}<.05$; Percentages exclude missing data. 


\subsection{Barriers to Engaging in SBECSA}

With respect to barriers, no items were considered applicable by $50 \%$ or more of the coaches (Table 3 ). Barriers that over $40 \%$ of the coaches perceived as applicable were as follows: b1, gap between a coaching ideal and actual experience (44.6\%); b11, no environment where coaches can work $(41.5 \%)$; b14, institutional limitation on working range $(44.6 \%)$; b27, insufficient contact with SBECSA teacher (41.6\%); b30, insufficient parenting (44.6\%); and b39, low motivation of team members $(40.9 \%)$. Poor financial compensation was not regarded as an important barrier (13.5\%).

Only one barrier-b31, poor relationships between parents and SBECSA teacher $(40.0 \%$ versus $18.0 \%$ ) - was reported more frequently by female than male. Coaches in the younger age-group (20-46 years) were significantly more likely to report barriers than their older counterparts (47-80 years), and they did so for 22 of the 54 items. Nine of these items were perceived to be applicable by more than $40 \%$ of the participants aged 20-46 years: b2, concerns about own coaching method (45.7\% versus $28.2 \%)$; b11, no environment where coaches can work (50.7\% versus $33.8 \%)$; b18, unsure of status or role $(41.4 \%$ versus $17.9 \%)$; b20, school policy that prohibits involvement of external coaches (45.7\% versus $28.6 \%)$; b21, teachers who have negative opinions about involvement of external coaches $(43.5 \%$ versus $23.4 \%$ ); b24, SBECSA teachers who rarely come to the field (41.4\% versus $19.2 \%)$; b27, insufficient contact with SBECSA teacher (51.4\% versus $32.1 \%$ ); b36, poor relationships with team members $(40.0 \%$ versus $23.1 \%)$; and $b 43$, inadequate facilities or equipment $(48.6 \%$ versus $30.8 \%$ ). Only one barrier - b38, retirement of external coach's own child from SBECSA (14.9\% versus $0.0 \%$ - was accorded a higher rate by the coaches aged over 47 years. There were no significant proportional differences among the barriers between junior high and high school.

Seven items showed a higher proportion among the coaches who were willing to become full-time teachers: b9, no credit on teacher adaptation examination (29.4\% versus $4.2 \%)$; b11, no environment where coaches can work $(75.0 \%$ versus $37.7 \%)$; b20, school policy that prohibits involvement of external coaches $(58.8 \%$ versus $33.6 \%)$; b24, SBECSA teachers who rarely come to the field $(58.8 \%$ versus $25.2 \%)$; b25, insufficient efforts to accept external coaches $(52.9 \%$ versus $20.5 \%$ ); b26, conflicting opinions with SBECSA teacher (58.8\% versus 20.3\%); and b28, lack of understanding from parents (47.1\% versus $21.3 \%$ ). However, one item-b52, unrealistic expectations from teachers or parents $(30.1 \%$ versus $0.0 \%$ ) - was accorded a higher rate by coaches who were not willing to become full-time teachers. 
Table 3. Percentages for barriers to involvement among the external coaches

\begin{tabular}{|c|c|c|c|c|c|c|c|}
\hline \multirow{2}{*}{$\begin{array}{l}\text { Large } \\
\text { category (6) }\end{array}$} & \multirow[t]{2}{*}{ Middle category (24) } & \multirow[t]{2}{*}{ Small category (54) } & \multirow[t]{2}{*}{ Overall } & \multicolumn{2}{|c|}{ Age group } & \multicolumn{2}{|c|}{$\begin{array}{l}\text { Willing to be a } \\
\text { teacher }\end{array}$} \\
\hline & & & & $20-46$ & $47-80$ & Yes & No \\
\hline \multirow{7}{*}{$\begin{array}{l}\text { negative } \\
\text { feelings }\end{array}$} & \multirow{3}{*}{$\begin{array}{l}\text { worries about } \\
\text { coaching }\end{array}$} & $\begin{array}{l}\text { b1. gap between a coaching } \\
\text { ideal and actual experience }\end{array}$ & 44.6 & 45.7 & 44.2 & 29.4 & 45.1 \\
\hline & & $\begin{array}{l}\text { b2. concerns about own } \\
\text { coaching method }\end{array}$ & 36.9 & $45.7 *$ & 28.2 & 52.9 & 34.1 \\
\hline & & $\begin{array}{l}\text { b3. having to engage in tasks } \\
\text { other than technical coaching }\end{array}$ & 32.7 & 30.4 & 35.1 & 17.6 & 33.1 \\
\hline & \multirow{2}{*}{$\begin{array}{l}\text { physical and mental } \\
\text { fatigue }\end{array}$} & b4. feeling fatigued & 18.8 & 24.3 & 12.8 & 17.6 & 16.3 \\
\hline & & $\begin{array}{l}\text { b5. poor competition score } \\
\text { for external coach }\end{array}$ & 15.0 & 18.6 & 11.8 & 17.6 & 14.8 \\
\hline & $\begin{array}{l}\text { concerns about team } \\
\text { members becoming } \\
\text { injured }\end{array}$ & $\begin{array}{l}\text { b6. concerns about team } \\
\text { members becoming injured }\end{array}$ & 26.2 & $37.1^{* *}$ & 16.7 & 17.6 & 26.0 \\
\hline & lack of enjoyment & $\begin{array}{l}\text { b7. not enjoying the } \\
\text { coaching experience }\end{array}$ & 36.1 & 42.0 & 29.9 & 41.2 & 35.0 \\
\hline \multirow{12}{*}{$\begin{array}{l}\text { inadequate } \\
\text { system }\end{array}$} & \multirow{5}{*}{ primitive system } & $\begin{array}{l}\text { b8. no mediation system for } \\
\text { external coaches }\end{array}$ & 21.5 & 25.7 & 17.9 & 17.6 & 21.1 \\
\hline & & $\begin{array}{l}\text { b9. no credit on teacher } \\
\text { adaptation examination }\end{array}$ & 6.9 & 8.6 & 5.4 & $29.4^{* *}$ & 4.2 \\
\hline & & $\begin{array}{l}\text { b10. no educational system } \\
\text { for external coaches }\end{array}$ & 33.3 & 40.6 & 26.0 & 47.1 & 32.2 \\
\hline & & $\begin{array}{l}\text { b11. no environment where } \\
\text { coaches can work }\end{array}$ & 41.5 & $50.7 *$ & 33.8 & $75.0 * *$ & 37.7 \\
\hline & & $\begin{array}{l}\text { b12. not continuing the } \\
\text { involvement of external } \\
\text { coaches }\end{array}$ & 19.7 & $27.5^{*}$ & 13.0 & 29.4 & 16.5 \\
\hline & \multirow{3}{*}{$\begin{array}{l}\text { limitations of the } \\
\text { system }\end{array}$} & $\begin{array}{l}\text { b13. institutional limitation } \\
\text { on coaching frequency }\end{array}$ & 23.5 & 30.0 & 17.9 & 35.3 & 20.3 \\
\hline & & $\begin{array}{l}\text { b14. institutional limitation } \\
\text { on working range }\end{array}$ & 44.6 & 50.0 & 40.3 & 47.1 & 43.4 \\
\hline & & $\begin{array}{l}\text { b15. institutional limitation } \\
\text { on number of external } \\
\text { coaches }\end{array}$ & 20.9 & $30.0 *$ & 13.0 & 29.4 & 18.0 \\
\hline & \multirow{2}{*}{$\begin{array}{l}\text { lack of } \\
\text { compensation }\end{array}$} & $\begin{array}{l}\text { b16. little or no } \\
\text { compensation }\end{array}$ & 13.5 & $20.0 *$ & 7.8 & 11.8 & 13.1 \\
\hline & & b17. not an hourly wage & 2.7 & 4.3 & 1.3 & 0.0 & 3.3 \\
\hline & \multirow[b]{2}{*}{ unclear status or role } & b18. unsure of status or role & 29.5 & $41.4^{* *}$ & 17.9 & 35.3 & 28.5 \\
\hline & & $\begin{array}{l}\text { b19. no official request from } \\
\text { school }\end{array}$ & 16.8 & 18.6 & 14.1 & 23.5 & 14.6 \\
\hline
\end{tabular}




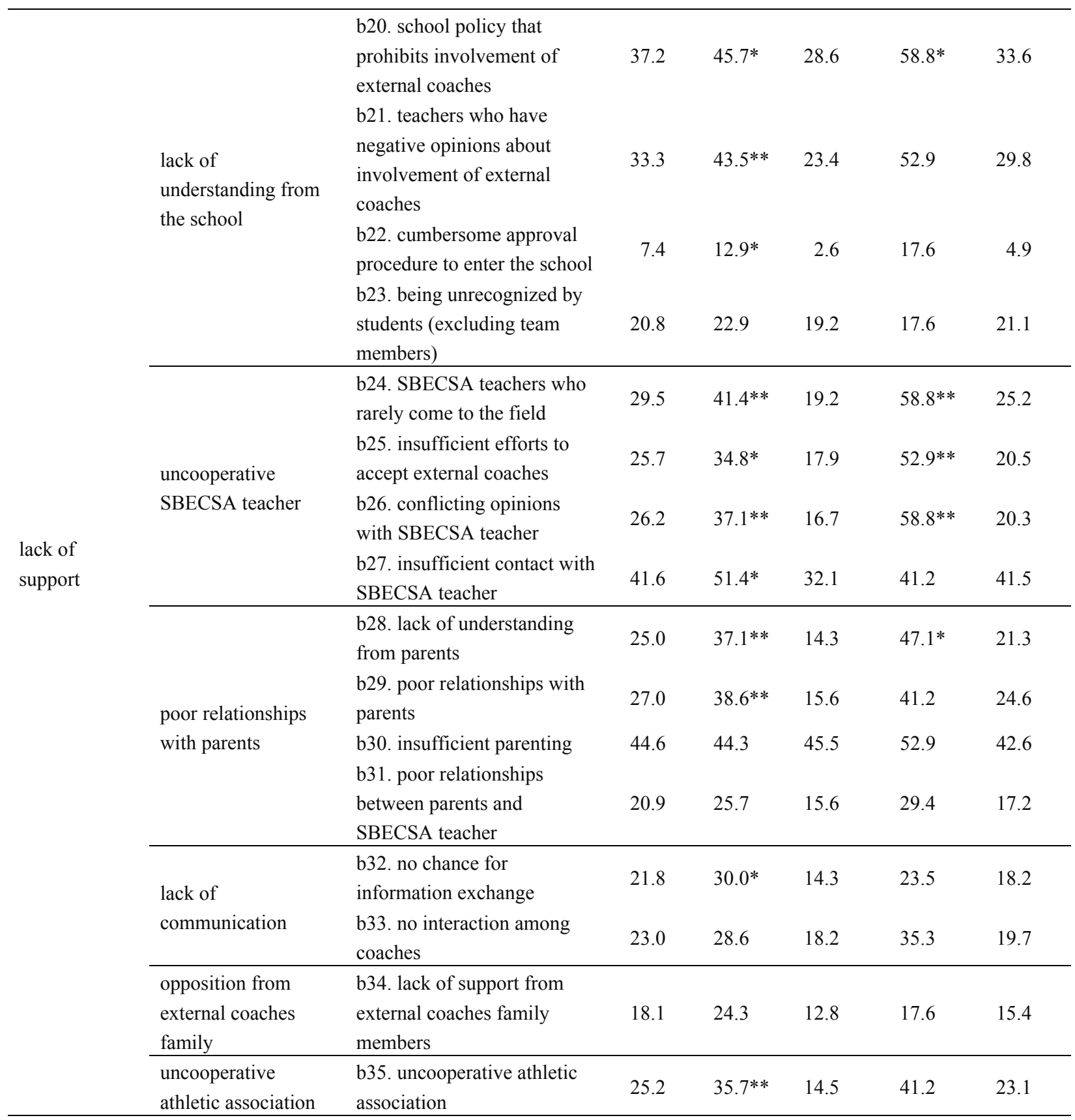




\begin{tabular}{|c|c|c|c|c|c|c|c|}
\hline \multirow{5}{*}{$\begin{array}{l}\text { uncomfortable } \\
\text { climate of } \\
\text { SBECSA }\end{array}$} & & $\begin{array}{l}\text { b36. poor relationships with } \\
\text { team members }\end{array}$ & 31.5 & $40.0^{*}$ & 23.1 & 41.2 & 29.3 \\
\hline & $\begin{array}{l}\text { poor relationships } \\
\text { with team members }\end{array}$ & $\begin{array}{l}\text { b37. having team members } \\
\text { who do not accept external } \\
\text { coach's instruction }\end{array}$ & 34.2 & 41.4 & 26.9 & 35.3 & 33.3 \\
\hline & & $\begin{array}{l}\text { b38. retirement of external } \\
\text { coach's own child from } \\
\text { SBECSA }\end{array}$ & 7.6 & 0.0 & $14.9 * *$ & 0.0 & 8.3 \\
\hline & $\begin{array}{l}\text { low motivation of } \\
\text { team members }\end{array}$ & $\begin{array}{l}\text { b39. low motivation of team } \\
\text { members }\end{array}$ & 40.9 & 45.7 & 37.2 & 41.2 & 40.7 \\
\hline & $\begin{array}{l}\text { despair or distress of } \\
\text { team members }\end{array}$ & $\begin{array}{l}\text { b40. despair or distress of } \\
\text { team members }\end{array}$ & 22.8 & 20.0 & 25.6 & 17.6 & 22.8 \\
\hline \multirow{4}{*}{$\begin{array}{l}\text { poor } \\
\text { environment }\end{array}$} & $\begin{array}{l}\text { inconvenient } \\
\text { practice time }\end{array}$ & $\begin{array}{l}\text { b41. inconvenient practice } \\
\text { time }\end{array}$ & 32.7 & 39.1 & 27.3 & 29.4 & 30.6 \\
\hline & $\begin{array}{l}\text { inconvenient } \\
\text { location }\end{array}$ & b42. long distance to school & 12.1 & 14.3 & 9.0 & 11.8 & 11.4 \\
\hline & $\begin{array}{l}\text { inadequate facilities } \\
\text { or equipment }\end{array}$ & $\begin{array}{l}\text { b43. inadequate facilities or } \\
\text { equipment }\end{array}$ & 39.6 & $48.6^{*}$ & 30.8 & 47.1 & 39.0 \\
\hline & bad weather & b44. bad weather & 7.5 & 8.6 & 6.6 & 0.0 & 8.3 \\
\hline \multirow{10}{*}{ burdens } & & b45. decrease in private time & 26.4 & $34.3 *$ & 19.5 & 23.5 & 26.2 \\
\hline & & b46. requires time & 20.1 & 25.7 & 15.4 & 23.5 & 19.5 \\
\hline & time burdens & $\begin{array}{l}\text { b47. no extra time to do } \\
\text { part-time job }\end{array}$ & 4.7 & 8.6 & 1.3 & 11.8 & 4.1 \\
\hline & & $\begin{array}{l}\text { b48. having to sacrifice } \\
\text { holidays }\end{array}$ & 24.0 & 30.0 & 18.7 & 11.8 & 25.6 \\
\hline & \multirow{4}{*}{$\begin{array}{l}\text { pressure or } \\
\text { expectations }\end{array}$} & $\begin{array}{l}\text { b49. having the } \\
\text { responsibility }\end{array}$ & 34.2 & 31.4 & 37.2 & 29.4 & 34.1 \\
\hline & & $\begin{array}{l}\text { b50. not finishing coaching } \\
\text { obligations before the next } \\
\text { coaching day }\end{array}$ & 19.6 & 22.9 & 15.6 & 23.5 & 19.7 \\
\hline & & $\begin{array}{l}\text { b51. pressure from alumni } \\
\text { organization }\end{array}$ & 18.2 & $28.6 * *$ & 7.8 & 23.5 & 17.2 \\
\hline & & $\begin{array}{l}\text { b52. unrealistic expectations } \\
\text { from teachers or parents }\end{array}$ & 28.2 & 27.1 & 29.5 & 0.0 & $30.1 * *$ \\
\hline & \multirow{2}{*}{$\begin{array}{l}\text { negative effect on } \\
\text { primary job }\end{array}$} & $\begin{array}{l}\text { b53. difficulty balancing } \\
\text { primary job and coaching }\end{array}$ & 27.7 & 32.9 & 23.4 & 29.4 & 26.2 \\
\hline & & $\begin{array}{l}\text { b54. having to compete } \\
\text { against other school teams }\end{array}$ & 7.4 & 5.7 & 9.0 & 0.0 & 8.1 \\
\hline
\end{tabular}

\section{Discussion}

A cross-sectional self-administrated questionnaire survey were conducted among external SBECSA coaches at junior high and high schools across Japan to examine the importance of facilitators and barriers in engaging the coaches. It was also aimed to clarify whether differences existed in perceptions of the survey items with respect to various characteristics of the coaches.

Most of the highly perceived (over 90\%) facilitators may be regarded as intrinsic motivation - doing something because it is inherently interesting or enjoyable (Ryan \& Deci, 
2000): $f 2$, feeling enjoyment; f5, feeling enjoyment to consider team members; f12, love of the sport that external coach plays; $\mathrm{f16}$, desire for team members to win; $\mathrm{f} 30$, seeing technical improvement in team members; $\mathrm{f} 31$, seeing personal progress in team members; $\mathrm{f} 40$, learning of external coach. By contrast, the facilitator related to the extrinsic motivation of financial compensation (Ryan \& Deci, 2000) was comparatively low (12.2\%). Previous surveys conducted among school principals indicated that prohibitive costs were a reason for not outsourcing external coaches (Williams et al., 2011), and greater compensation has been targeted (Miyagi Prefecture Board of Education, 2008). However, the results of the present study indicate that improving the environment in which external coaches can feel enjoyment or undergo personal development (i.e., intrinsic motivations) are more important than greater compensation.

In terms of barriers, b14 - institutional limitation on working range — was highly perceived (44.6\%). Some prefectures in Japan have rules whereby external coaches are not allowed to take students to competitions or out of school alone; they are also unable to attend managerial conferences (Hokkaido Junior High School Physical Culture Association, 2006, 2010). Easing such limitations would make external coaches engage more readily in SBECSA.

In the middle category of "uncooperative SBECSA teacher" indicated in Table 3, one item-b27, insufficient contact with SBECSA teacher-was a highly perceived barrier (41.6\%). Additionally, the large category of "comfortable climate of SBECSA" was more likely to be reported as a facilitator. As suggested by previous studies (Aoyagi et al., 2013b, 2013c), teachers should engage in SBECSA cooperatively and allot an appropriate role to the external coach.

A high proportion of participants in the younger age-group (20-46 years) reported worries about coaching (b2, concerns about own coaching method) and uncooperative SBECSA teachers (b24, SBECSA teachers who rarely come to the field; b27, insufficient contact with SBECSA teacher) as barriers. However, there are cases of teachers wanting to recruit young external coaches (Aoyagi et al., 2013a). Holding workshops toward eliminating worries about coaching and enhancing support from teachers would be valuable in encouraging the engagement of young coaches.

External coaches who were willing to become full-time teachers tended to coach toward gaining experience ( $\mathrm{f} 47$, accumulating coaching experience) and networking ( $\mathrm{f} 49$, being able to network; f50, increasing acquaintances), regardless of ease of access (f33, alma mater of external coach; f34, close proximity of school). Because teachers require that external coaches think as educators (Aoyagi et al., 2013a; Flintoff, Foster, \& Wystawnoha, 2011), coaches who want to become full-time teachers may be considered suitable human resources. Feedback from teachers is believed to enhance the experience of external coaches (National Institute of Fitness and Sports in Kanoya, Student Sports Volunteer Support Room, 2012). Workshops also have the potential to build up networks with other participants (Lydon \& King, 2009). Therefore, when targeting external coaches with educational thinking or knowledge, providing feedback or holding workshops could be useful strategies.

Although there were few differences among the facilitators and barriers by gender, female 
coaches more likely to perceive $\mathrm{f} 25$ (understanding from parents) as a facilitator and b31 (poor relationships between parents and SBECSA teacher) as a barrier. Creating a good relationship among the external coach, teacher, and parents would be supportive for female coaches. Beyond the question items that appeared in the present study, LaVoi and Dutove (2012) reviewed the barriers for female coaches, such as perceptions of being underpaid, limited upward career mobility, feelings of exclusion by male colleagues, and a lack of respect from athletes. An interview-based investigation (Aoyagi et al., 2013c), which supplied the basis for the questionnaire used in the present study and conducted among male and female Japanese external SBECSA coaches in junior high and high schools, did not identify the above barriers (LaVoi \& Dutove, 2012). Differences in the reported barriers may arise through the targeted school type (junior high and high school or university) or professional level (including compensation).

The present study found few differences between coaching in junior high and high schools. It is thus unnecessary to consider differences in school type when examining strategies to promote the recruitment of external SBECSA coaches. One study found a difference between primary and secondary schools: in light of the children's developmental stage, primary school teachers were more likely to require that external coaches possess an educational viewpoint than improve performance (Flintoff et al., 2011). A comparison of facilitators and barriers between coaches in elementary school and those in junior high and high schools may have yielded certain differences.

The current study has some limitations. First, although the participants were collected nationwide, the representativeness of participants may have been insufficient owing to the low response rate. Second, the analysis was cross-sectional, thereby making it impossible to identify the cause and effect. However, the cause-and-effect relationship would not make a large difference in the ultimate practice of SBECSA: enhancing facilitators and reducing barriers is certain to benefit external coaches and improve the SBECSA coaching environment. Despite these limitations, the present study adds quantitative support to the facilitators and barriers reported by Aoyagi et al. (2013c). Describing these facilitators and barriers with a quantitative method, such as the mixed-methods approach previously conceptualized by Creswell (2014), is important toward determining an effective strategy for a large number of external coaches. The results of the present study may be applied to improve the effectiveness of recruiting and managing external coaches.

\section{Conclusion}

In the present study, a cross-sectional self-administrated questionnaire was distributed to external coaches in order to evaluate the facilitators and barriers for engaging external coaches in school-based extracurricular sports activities and to determine whether differences in facilitators and barriers exist for the coaches and if these are related to the various characteristics of the coaches. Most of the highly perceived facilitators may be regarded as intrinsic motivation; while the facilitator related to the extrinsic motivation of financial compensation was low perception. In terms of barriers, institutional limitation and 
uncooperative SBECSA teacher were highly perceived. Some differences were found in the responses related to the coaches' age-group and their willingness or unwillingness to become full-time teachers. Adapting strategies to reflect the most commonly perceived facilitators and barriers would be effective in promoting the engagement of external SBECSA coaches. In particular, policy makers and human resource organizations which mediate schools and coaches need to ease institutional limitations and hold SBECSA workshops. Additionally, SBECSA teachers should improve the coaching environment such that the coach can feel enjoyment or undergo personal development, support the coach and participate in the SBECSA, and allot appropriate roles for the external coach.

\section{Acknowledgement}

The authors would like to thank all participating external coaches and the individuals who introduced participants. The present study was supported by the Research Grant (No. 26-3450) from Japan Society for the Promotion of Science, and Global COE Program "Sport Sciences for the Promotion of Active Life" from the Ministry of Education, Culture, Sports, Science and Technology in Japan.

\section{References}

Aoyagi, K., Ishii, K., Shibata, A., Arai, H., Hibi, C., \& Oka, K. (2013a). Factors associated with teachers' recruitment and continuous engagement of external coaches in school-based extracurricular sports activities: A qualitative study. Advances in Physical Education, 3(2), 62-70. http://dx.doi.org/10.4236/ape.2013.32010

Aoyagi, K., Ishii, K., Shibata, A., Arai, H., Hibi, C., \& Oka, K. (2013b). Correlates of engagement in school-based extracurricular sports activities among registrants of sports leader banks. Journal of Physical Education and Sport, 13(2), 127-134. http://dx.doi.org/10.7752/jpes.2013.02021

Aoyagi, K., Ishii, K., Shibata, A., Arai, H., Hibi, C., \& Oka, K. (2013c). Facilitators and barriers of external coaches' involvement into school-based extracurricular sports activities: A qualitative study. Advances in Physical Education, 3(3), 116-124. http://dx.doi.org/10.4236/ape.2013.33020

Australian Bureau of Statistics. (2012). Children's participation in cultural and leisure activities, Australia, Apr 2012. Retrieved June 13, 2014 from http://www.abs.gov.au/ausstats/abs@.nsf/mf/4901.0

Barnett, L. A. (2007). "Winners" and "losers": The effects of being allowed or denied entry into competitive extracurricular activities. Journal of Leisure Research, 39(2), 316-344.

Creswell, J. W. (2014). Research design: Qualitative, quantitative, and mixed methods approaches. (4th ed.). Thousand Oaks, CA: Sage. 
Dotterer, A. M., McHale, S. M., \& Crouter, A. C. (2007). Implications of out-of-school activities for school engagement in African American adolescents. Journal of Youth and Adolescence, 36, 391-401. http://dx.doi.org/10.1007/s10964-006-9161-3

Edwards, M. B., Kanters, M. A., \& Bocarro, J. N. (2011). Opportunities for extracurricular physical activity in North Carolina middle schools. Journal of Physical Activity and Health, 8, 597-605.

Flintoff, A., Foster, R., \& Wystawnoha, S. (2011). Promoting and sustaining high quality physical education and school sport through school sport partnerships. European Physical Education Review, 341-351. http://dx.doi.org/10.1080/13573320802445017

Fredricks, J. A., \& Eccles, J. S. (2006). Is extracurricular participation associated with beneficial outcomes? Concurrent and longitudinal relations. Developmental Psychology, 42(4), 698-713. http://dx.doi.org/10.1037/0012-1649.42.4.698

Hokkaido Junior High School Physical Culture Association. (2006). As regards external coach in Hokkaido junior high school athletic meeting.

Hokkaido Junior High School Physical Culture Association. (2010). Rights of external coaches in Hokkaido junior high school athletic meeting.

Japan Senior High School Teachers and Staff Union. (2008). Final report of actual condition survey for issues of school-based extracurricular sport activity in 2006.

Kanagawa Prefecture Board of Education. (2008). Research report for sports activity of $\begin{array}{llll}\text { secondary school student. } & \text { Retrieved } & \text { from }\end{array}$ http://www.pref.kanagawa.jp/uploaded/attachment/176796.pdf (June 13, 2014).

LaVoi, M. N., \& Dutove, K. J. (2012). Barriers and supports for female coaches: An ecological model. Sports Coaching Review, 1(1), 17-37. http://dx.doi.org/10.1080/21640629.2012.695891

Lipscomb, S. (2007). Secondary school extracurricular involvement and academic achievement: A fixed effects approach. Economics of Education Review, 26, 463-472. http://dx.doi.org/10.1016/j.econedurev.2006.02.006

Lydon, S., \& King, C. (2009). Can a single, short continuing professional development workshop cause change in the classroom? Professional Development in Education, 35(1), 63-82. http://dx.doi.org/10.1080/13674580802264746

Meester, A. D., Aelterman, N., Cardon, G., Bourdeaudhuij, I. D., \& Haerens, L. (2014). Extracurricular school-based sports as a motivating vehicle for sports participation in youth: A cross-sectional study. International Journal of Behavioral Nutrition and Physical Activity, 11(48). http://dx.doi.org/10.1186/1479-5868-11-48

Ministry of Education, Culture, Sports, Science and Technology in Japan. (1997). Report of investigative research for way of school-based extracurricular sport activity. Retrieved 
from

June

13

2014

http://www.mext.go.jp/b_menu/shingi/chousa/sports/001/toushin/971201.htm

Ministry of Education, Culture, Sports, Science and Technology in Japan. (2008). The course of study in junior high school. Retrieved June 13, 2014 from http://www.mext.go.jp/a_menu/shotou/new-cs/youryou/1304424.htm

Ministry of Education, Culture, Sports, Science and Technology in Japan. (2009). The course of study in higher school. Retrieved June 13, 2014 from http://www.mext.go.jp/a_menu/shotou/new-cs/youryou/1304427.htm

Ministry of Education, Culture, Sports, Science and Technology in Japan. (2011). Basic law of sports. $\quad$ Retrieved June 13, 2014 from http://www.mext.go.jp/a_menu/sports/kihonhou/attach/1307658.htm

Ministry of Education, Culture, Sports, Science and Technology in Japan. (2012). National survey result of physical and athletic capacity. Retrieved June 13, 2014 from http://www.mext.go.jp/component/b_menu/other/_icsFiles/afieldfile/2012/10/09/13265 91_07.pdf

Ministry of Education, Culture, Sports, Science and Technology in Japan. (2013). Report of investigative research for way of school-based extracurricular sport activity: Aim for school-based extracurricular sport activity that every student can bright. Retrieved June 13, 2014 from http://www.mext.go.jp/a_menu/sports/jyujitsu/_icsFiles/afieldfile/2013/05/27/1335529 -1.pdf

Miyagi Prefecture Board of Education. (2008). Research for school-based extracurricular sport activity of secondary school.

National Institute of Fitness and Sports in Kanoya, Student Sports Volunteer Support Room. (2012). Guide book for student sports volunteer coaches. Kagoshima, Japan: National Institute of Fitness and Sports in Kanoya, Student Sports Volunteer Support Room.

Nippon Junior High School Physical Culture Association. (2013). Spread sheet of research for number of member school and student. Retrieved June 13, 2014 from http://www18.ocn.ne.jp/ njpa/kamei.html

Nishijima, H., Yano, H., \& Nakazawa, A. (2007). A sociological study of coaching and management of club activities in junior high schools: Based on a questionnaire survey to teachers of sports club activities in two prefectures and Tokyo metropolitan. Bulletin of Faculty of Education in the University of Tokyo, 47, 101-130.

Ryan, R.M., \& Deci, E.L. (2000). Intrinsic and extrinsic motivations: Classic definitions and new directions. Contemporary Educational Psychology, 25, 54-67. http://dx.doi.org/10.1006/ceps.1999.1020

Sasakawa Sports Foundation. (2011). Sports white paper: Future that sports should to aspire. Tokyo, Japan: Sasakawa Sports Foundation. 
Schaefer, D. R., Simpkins, S. D., Vest, A. E., \& Price, C. D. (2011). The contribution of extracurricular activities to adolescent friendships: New insights through social network. Developmental Psychology, 47(4), 1141-1152. http://dx.doi.org/10.1037/a0024091

Shernoff, D. J., \& Vandell, D. L. (2007). Engagement in after-school program activities: Quality of experience from the perspective of participants. Journal of Youth and Adolescence, 36, 891-903. http://dx.doi.org/10.1007/s10964-007-9183-5

Sport Council Wales. (2009). Young people's participation in sport. Sportsupdate, 62, 11-16.

Telama, R. (2009). Tracking of physical activity from childhood to adulthood: A review. Obesity Facts, 3, 187-195. http://dx.doi.org/10.1249/01.MSS.0000145525.29140.3B

Tokyo Metropolitan Board of Education. (2007). Handbook for teachers who manage school-based extracurricular activity. Retrieved June 13, 2014 from http://www.kyoiku.metro.tokyo.jp/buka/shidou/bukatsudo/pr070412h/pr070412_hb.pdf

Tokyo Metropolitan Board of Education. (2008). Companion of coaching school-based extracurricular activity for external coach. Retrieved June 13, 2014 from http://www.kyoiku.metro.tokyo.jp/press/bukatsu_tebiki.pdf

Whiteley, R. F., \& Richard, G. (2012). Timetabling and extracurricular activities: A study of teachers' attitudes towards preparation time. Management in Education, 26(1), 6-12. http://dx.doi.org/10.1177/0892020611426894

Williams, J. B., Hay, J. P., \& Macdonald, D. (2011). The outsourcing of health, sport and physical educational work: A state of play. Physical Education and Sport Pedagogy, 16(4), 399-415. http://dx.doi.org/10.1080/17408989.2011.582492

Yamagata Prefecture Board of Education. (2010). As regards a way of future school-based extracurricular sport activity. Retrieved March 11, 2014 from http://www.pref.yamagata.jp/ou/kyoiku/700021/gakutai-toppage/21 unndoubukatudouno arikata.html

\section{Copyright Disclaimer}

Copyright for this article is retained by the author(s), with first publication rights granted to the journal.

This is an open-access article distributed under the terms and conditions of the Creative Commons Attribution license (http://creativecommons.org/licenses/by/3.0/). 